\title{
EORTC Nomograms and Risk Groups for Predicting Recurrence, Progression, and Disease-specific and Overall Survival in Non-Muscle-invasive Stage Ta-T1 Urothelial Bladder Cancer Patients Treated with 1-3 Years of Maintenance Bacillus Calmette-Guérin
}

\author{
Samantha Cambier ${ }^{a, \dagger}$, Richard J. Sylvester ${ }^{a, *}$, Laurence Collette ${ }^{a}$, Paolo Gontero ${ }^{b}$, \\ Maurizio A. Brausi ${ }^{c}$, George van Andel ${ }^{d}$, Wim J. Kirkels ${ }^{e}$, Fernando Calais Da Silva ${ }^{f}$, \\ Willem Oosterlinck ${ }^{g}$, Stephen Prescott ${ }^{h}$, Ziya Kirkali ${ }^{i \ddagger}$, Philip H. Powell ${ }^{j}$, Theo M. de Reijke ${ }^{k}$, \\ Levent Turkeri ${ }^{l}$, Sandra Collette ${ }^{a}$, Jorg Oddens ${ }^{m}$

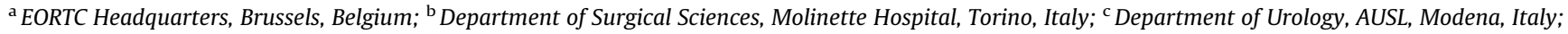 \\ ${ }^{\mathrm{d}}$ Department of Urology, OLVG, Amsterdam, The Netherlands; ${ }^{\mathrm{e}}$ Department of Urology, Erasmus Medical Center, Rotterdam, The Netherlands; ${ }^{\mathrm{f}}$ Department \\ of Urology, Hospital Do Desterro, Lisbon, Portugal; ${ }^{g}$ Department of Urology, Ghent University Hospital, Ghent, Belgium; ${ }^{\mathrm{h}}$ Department of Urology, St. James's \\ University Hospital, Leeds, UK; ${ }^{\mathrm{i}}$ Department of Urology, Dokuz Eylul University School of Medicine, Izmir, Turkey; ${ }^{\mathrm{j}}$ Department of Urology, Freeman Hospital, \\ Newcastle Upon Tyne, UK; ${ }^{k}$ Department of Urology, Academic Medical Center, Amsterdam, The Netherlands; ${ }^{1}$ Department of Urology, Marmara University \\ School of Medicine, Istanbul, Turkey; ${ }^{\mathrm{m}}$ Department of Urology, Jeroen Bosch Ziekenhuis, s'-Hertogenbosch, The Netherlands
}

\section{Article info}

Article history:

Accepted June 29, 2015

Associate Editor:

Giacomo Novara

\section{Keywords:}

Non-muscle-invasive bladder

cancer

Prognostic factors

Recurrence

Progression

Survival

Bacillus Calmette-Guérin

\begin{abstract}
Background: There are no prognostic factor publications on stage Ta-T1 non-muscleinvasive bladder cancer (NMIBC) treated with 1-3 yr of maintenance bacillus CalmetteGuérin (BCG).

Objective: To determine prognostic factors in NMIBC patients treated with 1-3 yr of BCG after transurethral resection of the bladder (TURB), to derive nomograms and risk groups, and to identify high-risk patients who should be considered for early cystectomy.

Design, setting, and participants: Data for 1812 patients were merged from two European Organization for Research and Treatment of Cancer randomized phase 3 trials in intermediate- and high-risk NMIBC.

Intervention: Patients received 1-3 yr of maintenance BCG after TURB and induction BCG. Outcome measurements and statistical analysis: Prognostic factors for risk of early recurrence and times to late recurrence, progression, and death were identified in a training data set using multivariable models and applied to a validation data set.

Results and limitations: With a median follow-up of $7.4 \mathrm{yr}, 762$ patients recurred; 173 progressed; and 520 died, 83 due to bladder cancer (BCa). Statistically significant prognostic factors identified by multivariable analyses were prior recurrence rate and number of tumors for recurrence, and tumor stage and grade for progression and death due to BCa. T1G3 patients do poorly, with 1- and 5-yr disease-progression rates of $11.4 \%$

Current address: Saint-Luc University Hospital, Brussels, Belgium

${ }^{\ddagger}$ Current address: National Institute of Diabetes and Digestive and Kidney Diseases, National Institutes of Health, Bethesda, MD, USA.

* Corresponding author. EORTC Headquarters, 83 avenue E. Mounier, Bte 11, 1200 Brussels, Belgium. Tel. +322 2741613 .

E-mail addresses: richard.sylvester@eortc.be, richard.sylvester@skynet.be (R.J. Sylvester).
\end{abstract}

http://dx.doi.org/10.1016/j.eururo.2015.06.045

0302-2838/C 2015 European Association of Urology. Published by Elsevier B.V. All rights reserved. 


\author{
EU * ACME \\ www.eu-acme.org/ \\ europeanurology \\ Please visit \\ www.eu-acme.org/ \\ europeanurology to read and \\ answer questions on-line. \\ The EU-ACME credits will \\ then be attributed \\ automatically.
}

and $19.8 \%$, respectively, and $1-$ and 5 -yr disease-specific death rates of $4.8 \%$ and $11.3 \%$. Limitations include lack of repeat transurethral resection in high-risk patients and exclusion of patients with carcinoma in situ.

Conclusions: NMIBC patients treated with 1-3 yr of maintenance BCG have a heterogeneous prognosis. Patients at high risk of recurrence and/or progression do poorly on currently recommended maintenance schedules. Alternative treatments are urgently required.

Patient summary: Non-muscle-invasive bladder cancer patients at high risk of recurrence and/or progression do poorly on currently recommended bacillus CalmetteGuérin maintenance schedules, and alternative treatments are urgently required.

Trial registration: Study 30911 was registered with the US National Cancer Institute clinical trials database (protocol ID: EORTC 30911). Study 30962 was registered at ClinicalTrials.gov, number NCT00002990; http://clinicaltrials.gov/ct2/show/record/ NCT00002990.

(C) 2015 European Association of Urology. Published by Elsevier B.V. All rights reserved.

\section{Introduction}

European Association of Urology (EAU) recommendations for adjuvant treatment of non-muscle-invasive bladder cancer (NMIBC) are based on low-, intermediate-, and highrisk groups [1,2]. They are derived from European Organization for Research and Treatment of Cancer (EORTC) risk tables, which provide probabilities of recurrence and progression after transurethral resection of the bladder; however, none of these patients received maintenance bacillus Calmette-Guérin (BCG), which is now recommended for high-risk patients [3].

In 1062 patients treated with a maintenance schedule of six two-weekly BCG instillations for 5-6 mo, the Club Urológico Español de Tratamiento Oncológico (CUETO) found that EORTC risk tables overestimated the risk of recurrence and probability of progression in high-risk patients [4]. CUETO stratified patients according to their risk of recurrence and progression [5,6], which also stratified a patient's risk of recurrence after BCG plus interferon- $\alpha$ therapy [7].

The CUETO maintenance schedule is considerably shorter than the 1-3 yr of maintenance BCG currently recommended by the EAU $[1,2]$. Prognostic factors for this longer maintenance schedule are unknown.

Our aim was to determine prognostic factors and the probabilities of recurrence, progression, disease-specific survival (DSS), and overall survival (OS) in stage Ta-T1 NMIBC patients treated with 1-3 yr of maintenance BCG and identify high-risk patients who may need early cystectomy. We also assessed the performance of CUETO models on EORTC data.

\section{Materials and methods}

Individual patient data were merged from two EORTC phase 3 trials (30911 and 30962) in Ta-T1 NMIBC (without carcinoma in situ [CIS]). Patients were randomized to receive 1 or $3 \mathrm{yr}$ of maintenance Tice BCG $[8,9]$ (Supplement 1). Treatment duration had no impact on determination of the prognostic factors. Its possible impact was diluted because $40-50 \%$ of patients randomized to $3 \mathrm{yr}$ of maintenance in the two studies received $\leq 1 \mathrm{yr}$, mainly due to inefficacy.

Patient and tumor characteristics previously investigated by EORTC or CUETO were retrospectively analyzed: age, gender, prior recurrence rate, number of tumors, largest tumor diameter, tumor stage, and 1973 World Health Organization (WHO) grade. The following end points were assessed.

Time to first recurrence (disease-free interval), defined as (1) early recurrence within the first 4.5 mo after randomization (time between randomization and first follow-up cystoscopy, normally at $3 \mathrm{mo}$, which was delayed in some patients) or (2) time to late recurrence (first recurrence in patients without an early recurrence who had follow-up after 4.5 mo [Landmark analysis]). Patients still alive without recurrence were censored at the last follow-up. The time was censored at death and/or cystectomy in the absence of recurrence (competing risk).

Time to progression was defined as the time from randomization to first increase to stage T2 or higher or distant metastases. Patients still alive without progression were censored at the last follow-up. The time was censored upon death and/or cystectomy before progression (competing risks).

Duration of survival was defined as the time from randomization to death from any cause or last follow-up. For DSS, deaths not due to bladder cancer (BCa) are competing risks. For both end points, patients still alive were censored at the last follow-up.

Median follow-up in all patients was estimated using the KaplanMeier technique and OS time but with status indicator reversed: Patients still alive were considered to have died at last follow-up, and patients who died were censored at date of death [10].

The data set was split into two sets based on country: a training set from the largest participating countries for model development and a validation set (Supplementary Table 1). Using previous publications as a guide [3,5,6], variables were coded based on training set univariate analyses.

In multivariable regression models for recurrence, variables were age (continuous), gender, prior recurrence rate (primary, less than or equal to one recurrence per year, or more than one recurrence per year), tumor size ( $<3 \mathrm{~cm}$ or $\geq 3 \mathrm{~cm}$ ), number of tumors ( $<4$ or $\geq 4$ [6]), stage (Ta or T1), and 1973 WHO grade (G1 or G2-G3). For progression, death due to BCa and OS, the same variables and coding were used except for tumor size (continuous), number of tumors ( $<8$ or $\geq 8$ ), and grade (G1, G2, or G3).

For early recurrence, multivariable logistic regression models were fit using Bayesian information criterion (BIC) variable selection to allow covariates to leave the model [11].

For competing risk end points, Fine and Gray models were fit using Bayesian information criterion for competing risks (BICcr) variable selection [12]. For OS, Cox models were fit based on BIC variable selection. Final models were based on the frequency of variables retained using BIC/BICcr selection procedures with bootstrap resampling.

Kaplan-Meier OS curves were estimated. Based on the multivariable model, a nomogram was constructed to predict 1- and 5-yr survival. The nomogram provides a graphic representation linking an individual patient's multivariable prognostic factors to his or her survival 
probability. Goodness of fit was assessed with calibration plots. For other end points, time-to-event distributions were estimated using cumulative incidence curves.

Based on multivariable analyses in the training set, prognostic factors were identified and prognostic categories formed. Categories with similar prognoses were grouped together, with emphasis placed on identifying the best prognosis and the worst prognosis patients. These categories and survival regression coefficients were applied to the validation set.

Area under the curve (AUC) and Harrell's bias-corrected concordance index (C-index) were used to assess model accuracy (discrimination) in the training and validation data sets $[13,14]$. The C-index is the probability that for two randomly chosen patients, the patient who had the event first has a higher probability of having the event according to the model. Consequently, $\mathrm{C}=0.50$ represents agreement by chance; $C=1.0$ represents perfect discrimination.

For all end points, sensitivity analyses were done stratified by study. Sensitivity analyses also compared results of the Cox and the Fine and Gray models.

Statistical analyses were done using SAS v.9.4 (SAS Institute, Cary, NC, USA) and R v.3.0.1 (R Foundation, Vienna, Austria) with crrstep package v.2014-07.16, cmprsk package v.2.2-7, and rms package v.4.2-1 for constructing nomograms.

\section{Results}

A total of 1812 Ta-T1 patients from EORTC studies 30962 $(n=1272$ patients) and $30911(n=540)$ were included, 1180 (65\%) of whom were allocated to $3 \mathrm{yr}$ of maintenance BCG and $632(35 \%)$ to $1 \mathrm{yr}$. They were divided into training and validation data sets with 1178 and 634 patients, respectively.

Median age was $67 \mathrm{yr}, 43 \%$ were recurrent, $24 \%$ had a prior recurrence rate of more than one per year, $87 \%$ had multiple tumors, $20 \%$ had tumors $\geq 3 \mathrm{~cm}, 36 \%$ were T1, and 23\% had G3 tumors (Table 1). Patient characteristics in the training and validation data sets were similar, except for a higher percentage of $\mathrm{T} 1$ patients in the validation data set ( $46 \%$ vs $31 \%$ ).

After a median follow-up of $7.4 \mathrm{yr}, 6.7 \mathrm{yr}$ in patients still alive, 762 patients recurred, 173 progressed, and 520 died, 83 due to BCa (Table 2).

The 1-and 5-yr recurrence rates were 25.9\% (95\% confidence interval [CI], 23.8-27.9\%) and 41.3\% (95\% CI, 39.0-43.7\%), respectively (Supplementary Fig. 1).

Overall, 285 patients (15.7\%) had an early recurrence (Table 2). Using the training data set, the final model for early recurrence included prior recurrence rate, number of tumors, and grade. Table 3 shows the probabilities of early recurrence in the training and validation data sets in the six prognostic groups. The best prognosis patients, G1 with fewer than four tumors and prior recurrence rate of less than or equal to one per year, had an $8 \%$ probability of early recurrence in the validation set. The worst prognosis group, four or more $\mathrm{G} 2$ or $\mathrm{G} 3$ tumors and more than one recurrence per year, had a $29 \%$ probability of early recurrence. For these six groups, the AUCs were 0.67 and 0.65 in the training and validation data sets, respectively.

Late recurrence was observed in 477 patients (Table 2). A total of 375 patients were excluded from the analysis, 285 due to early recurrence and 90 due to no follow-up after
Table 1 - Patient characteristics

\begin{tabular}{|c|c|c|c|}
\hline & $\begin{array}{c}\text { Training } \\
\begin{array}{c}(n=1178) \\
n(\%)\end{array}\end{array}$ & $\begin{array}{c}\text { Validation } \\
\begin{array}{c}(n=634) \\
n(\%)\end{array}\end{array}$ & $\begin{array}{c}\text { Total } \\
(n=1812) \\
n(\%)\end{array}$ \\
\hline \multicolumn{4}{|l|}{ Age, yr } \\
\hline$\leq 60$ & $333(28)$ & $162(26)$ & $495(27)$ \\
\hline $61-70$ & 397 (34) & $233(37)$ & $630(35)$ \\
\hline $71-80$ & $393(33)$ & $201(32)$ & $594(33)$ \\
\hline$>80$ & $55(4.7)$ & $38(6.0)$ & $93(5.1)$ \\
\hline \multicolumn{4}{|l|}{ Gender } \\
\hline Female & 199 (17) & $101(16)$ & $300(17)$ \\
\hline Male & $979(83)$ & $530(84)$ & $1509(83)$ \\
\hline Unknown & $0(0.0)$ & $3(0.5)$ & $3(0.2)$ \\
\hline \multicolumn{4}{|l|}{ Prior recurrence rate } \\
\hline Primary & $635(54)$ & $358(56)$ & $993(55)$ \\
\hline Recurrent, $\leq 1 / \mathrm{yr}$ & 220 (19) & 121 (19) & 341 (19) \\
\hline Recurrent, >1/yr & 297 (25) & $137(22)$ & $434(24)$ \\
\hline Unknown & $26(2.2)$ & $18(2.8)$ & $44(2.4)$ \\
\hline \multicolumn{4}{|l|}{ Largest tumor size } \\
\hline$\leq 1 \mathrm{~cm}$ & $517(44)$ & $306(48)$ & $823(45)$ \\
\hline$>1 \mathrm{~cm}$ to $<3 \mathrm{~cm}$ & $354(30)$ & $196(31)$ & $550(30)$ \\
\hline$\geq 3 \mathrm{~cm}$ & $247(21)$ & $112(18)$ & $359(20)$ \\
\hline Unknown & $60(5.1)$ & $20(3.2)$ & $80(4.4)$ \\
\hline \multicolumn{4}{|l|}{ No. of tumors } \\
\hline 1 & $175(15)$ & $69(11)$ & $244(13)$ \\
\hline $2-3$ & 700 (59) & $372(59)$ & $1072(59)$ \\
\hline $4-7$ & $253(21)$ & $138(22)$ & $391(22)$ \\
\hline$\geq 8$ & $50(4.2)$ & $55(8.7)$ & $105(5.8)$ \\
\hline \multicolumn{4}{|l|}{$\mathrm{T}$ category } \\
\hline Тa & 807 (69) & $345(54)$ & $1152(64)$ \\
\hline T1 & $370(31)$ & $289(46)$ & $659(36)$ \\
\hline Unknown & $1(0.1)$ & $0(0.0)$ & $1(0.1)$ \\
\hline \multicolumn{4}{|l|}{1973 WHO grade } \\
\hline G1 & 389 (33) & $191(30)$ & $580(32)$ \\
\hline G2 & $506(43)$ & 309 (49) & $815(45)$ \\
\hline G3 & $280(24)$ & $132(21)$ & $412(23)$ \\
\hline Unknown & $3(0.3)$ & $2(0.3)$ & $5(0.3)$ \\
\hline
\end{tabular}

the landmark. Prognostic variables in the final model were prior recurrence rate and number of tumors. Four prognostic groups were created (Table 4). The best prognostic group, fewer than four tumors and less than or equal to one recurrence per year, had recurrence probabilities of $14.0 \%$ and $28.3 \%$ at $1 \mathrm{yr}$ and $5 \mathrm{yr}$, respectively, in the validation set. The worst prognostic group, more than one recurrence per year and four or more tumors, had recurrence probabilities of $33.0 \%$ and $51.7 \%$ at 1 and $5 \mathrm{yr}$, respectively. The C-indexes in the training and validation data sets were 0.59 and 0.56 , respectively. Cumulative incidence curves are provided in Figure $1 \mathrm{a}$ and $1 \mathrm{~b}$.

Table 2 - Number of events

\begin{tabular}{llcl}
\hline & $\begin{array}{c}\text { Training } \\
(n=1178) \\
n(\%)\end{array}$ & $\begin{array}{c}\text { Validation } \\
(n=634) \\
n(\%)\end{array}$ & $\begin{array}{c}\text { Total } \\
(n=1812) \\
n(\%)\end{array}$ \\
\hline Recurrence & 509 & 253 & 762 \\
Early recurrence & $202(17.1)$ & $83(13.1)$ & $285(15.7)$ \\
\hline Late recurrence & 307 & 170 & 477 \\
Progression & 116 & 57 & 173 \\
Dead & 318 & 202 & 520 \\
\hline Bladder cancer & 59 & 24 & 83 \\
\hline Dead other cause & 259 & 178 & 437 \\
\hline
\end{tabular}




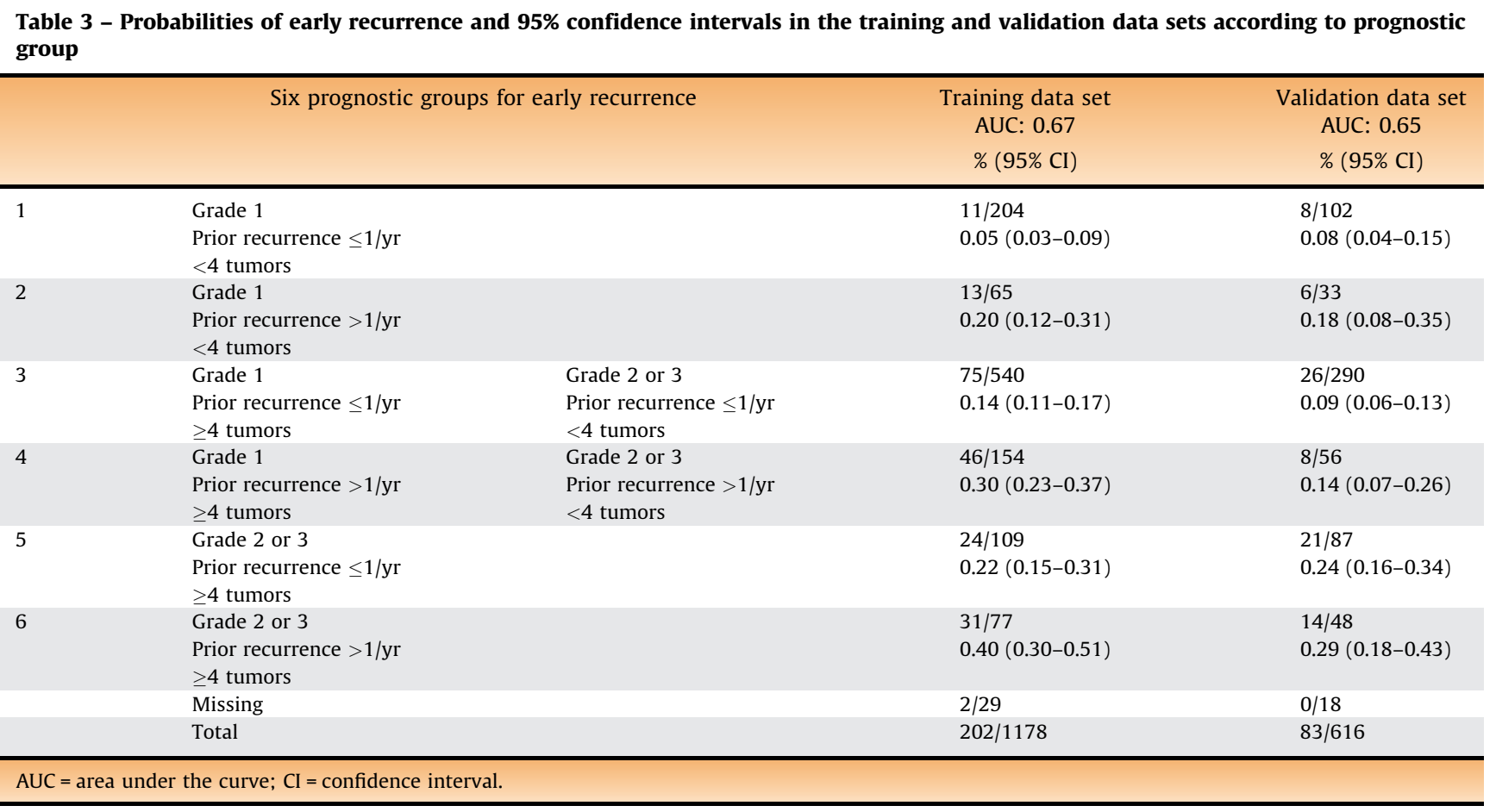

The multivariable model for disease progression identified two variables: grade and $\mathrm{T}$ category, with four prognostic groups (Table 5). The best prognostic group, TaG1 patients, had a progression probability of $1.9 \%$ at $1 \mathrm{yr}$ and $7.1 \%$ at $5 \mathrm{yr}$ in the validation data set. The worst prognostic group, T1G3 patients, had a progression probability of $11.4 \%$ at $1 \mathrm{yr}$ and $19.8 \%$ at $5 \mathrm{yr}$ (Table 5; Fig. 2a and $2 \mathrm{~b}$ ). The $\mathrm{C}$-indexes in the training and validation data sets were 0.72 and 0.64 , respectively.

A total of 83 of 520 deaths were due to BCa. The same variables as for progression were of prognostic importance, grade and $\mathrm{T}$ category, and the same prognostic groups were formed (Table 6). TaG1, the best prognostic group, had a probability of death due to BCa of $1.5 \%$ at $5 \mathrm{yr}$ in the validation set. The worst prognostic group, T1G3, had a probability of death due to BCa of $4.8 \%$ at $1 \mathrm{yr}$ and $11.3 \%$ at $5 \mathrm{yr}$ (Table 6 ). The $\mathrm{C}$-indexes in the training and validation data sets were 0.72 and 0.71 , respectively. Cumulative incidence curves are given in Figure $3 \mathrm{a}$ and $3 \mathrm{~b}$.
The final multivariable survival model included age and grade. The $\mathrm{C}$-indexes in the training and validation data sets were both 0.68 . Model calibration was checked with calibration plots (Supplementary Fig. 2a and 2b). Supplementary Figure 3a, 3b, 4a, and 4b present KaplanMeier curves according to age and grade, respectively, in both data sets. Figure 4 provides a nomogram for survival based on age and grade. Points associated with the nomogram are given in Supplementary Table 2. For example, a patient aged 65 yr with a G3 tumor has 79 points $(67+12)$, with survival probabilities of $97 \%$ at $1 \mathrm{yr}$ and $78 \%$ at $5 \mathrm{yr}$. Sensitivity analyses offered the same conclusions for all end points.

CUETO recurrence and progression scores were calculated on EORTC data [6]. Time to recurrence and time to progression cumulative incidence curves were estimated (Supplementary Fig. 5a and 5b). The CUETO model did not perform as well when applied to EORTC data. The C-indexes for recurrence and progression decreased from 0.64 to 0.48 and from 0.69 to 0.53 , respectively. Applied to EORTC

Table 4 - Probabilities of late recurrence and 95\% confidence intervals in the training and validation data sets according to prognostic group

\begin{tabular}{|c|c|c|c|c|}
\hline & \multicolumn{2}{|c|}{ Training data set: C-index 0.59} & \multicolumn{2}{|c|}{ Validation data set: C-index 0.56} \\
\hline & $1 \mathrm{yr}, \%(95 \% \mathrm{CI})$ & $5 \mathrm{yr}, \%(95 \% \mathrm{CI})$ & $1 \mathrm{yr}, \%(95 \% \mathrm{CI})$ & 5 yr, \% (95\% CI) \\
\hline $\begin{array}{l}\text { Prior recurrence } \leq 1 / \mathrm{yr} \\
\text { and }<4 \text { tumors }\end{array}$ & $\begin{array}{l}11.0 \\
(8.4-13.5)\end{array}$ & $\begin{array}{l}25.9 \\
(22.1-29.6)\end{array}$ & $\begin{array}{l}14.0 \\
(10.2-17.9)\end{array}$ & $\begin{array}{l}28.3 \\
(23.1-33.6)\end{array}$ \\
\hline $\begin{array}{l}\text { Prior recurrence } \leq 1 / \mathrm{yr} \\
\text { and } \geq 4 \text { tumors }\end{array}$ & $\begin{array}{l}20.6 \\
(13.9-27.3)\end{array}$ & $\begin{array}{l}31.6 \\
(23.7-39.5)\end{array}$ & $\begin{array}{l}16.3 \\
(8.5-24.1)\end{array}$ & $\begin{array}{l}31.0 \\
(20.8-41.3)\end{array}$ \\
\hline $\begin{array}{l}\text { Prior recurrence }>1 / y r \\
\text { and }<4 \text { tumors }\end{array}$ & $\begin{array}{l}23.8 \\
(16.5-31.2)\end{array}$ & $\begin{array}{l}39.6 \\
(30.9-48.2)\end{array}$ & $\begin{array}{l}21.8 \\
(11.3-32.3)\end{array}$ & $\begin{array}{l}42.4 \\
(29.3-55.5)\end{array}$ \\
\hline $\begin{array}{l}\text { Prior recurrence }>1 / y r \\
\text { and } \geq 4 \text { tumors }\end{array}$ & $\begin{array}{l}31.2 \\
(20.1-42.3)\end{array}$ & $\begin{array}{l}55.4 \\
(43.1-67.6)\end{array}$ & $\begin{array}{l}33.0 \\
(19.3-46.7)\end{array}$ & $\begin{array}{l}51.7 \\
(36.9-66.6)\end{array}$ \\
\hline
\end{tabular}



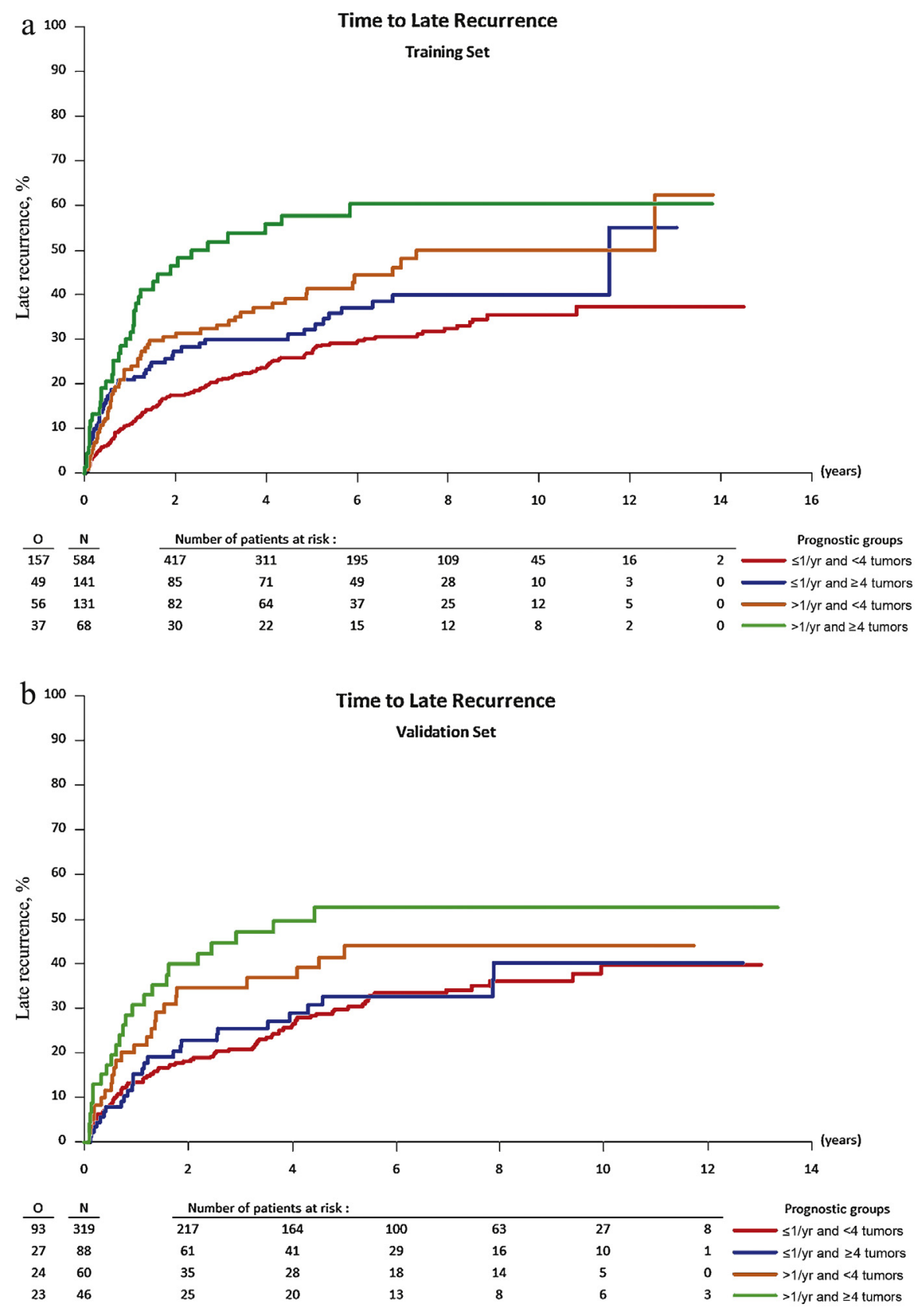

Fig. 1 - Time to late recurrence according to prognostic groups: (a) training set; (b) validation set.

data, the CUETO model underestimated the risk of recurrence in good risk patients and overestimated the risk of progression at $5 \mathrm{yr}$ in poor risk patients.

\section{Discussion}

This paper presents the first prognostic factor analysis in NMIBC patients receiving the currently recommended $1-3 \mathrm{yr}$ of maintenance BCG and identified prognostic factors for recurrence, progression, DSS, and OS.

The analysis made a distinction between early recurrence that may be influenced by unintended incomplete resection [15] and late recurrence in patients who are disease free at the first follow-up cystoscopy. The most important prognostic factors for early recurrence were prior recurrence rate, number of tumors, and grade. The prior recurrence rate and number of tumors were also the most important factors for time to recurrence in patients without early recurrence. These results correspond with those from the EORTC [3] and CUETO [5,6]. However, CUETO also identified the prognostic importance of age and gender.

For progression, the most important factors were stage and grade, which were also previously identified by EORTC 
Table 5 - Probabilities of progression and 95\% confidence intervals in the training and validation data sets according to stage and grade

\begin{tabular}{|c|c|c|c|c|}
\hline & \multicolumn{2}{|c|}{ Training data set: C-index 0.72} & \multicolumn{2}{|c|}{ Validation data set: C-index 0.64} \\
\hline & $1 \mathrm{yr}, \%(95 \% \mathrm{CI})$ & $5 \mathrm{yr}, \%(95 \% \mathrm{CI})$ & $1 \mathrm{yr}, \%(95 \% \mathrm{CI})$ & $5 \mathrm{yr}, \%(95 \% \mathrm{CI})$ \\
\hline TaG1 & $\begin{array}{l}0.28 \\
(0-0.82)\end{array}$ & $\begin{array}{l}2.4 \\
(0.76-4.1)\end{array}$ & $\begin{array}{l}1.9 \\
(0-4.1)\end{array}$ & $\begin{array}{l}7.1 \\
(2.8-11.4)\end{array}$ \\
\hline TaG2 or $\mathrm{T} 1 \mathrm{G} 1$ & $\begin{array}{l}2.5 \\
(0.97-4.0)\end{array}$ & $\begin{array}{l}7.0 \\
(4.4-9.6)\end{array}$ & $\begin{array}{l}1.6 \\
(0-3.4)\end{array}$ & $\begin{array}{l}4.6 \\
(1.5-7.7)\end{array}$ \\
\hline TaG3 or $\mathrm{T} 1 \mathrm{G} 2$ & $\begin{array}{l}3.5 \\
(0.75-6.3)\end{array}$ & $\begin{array}{l}15.2 \\
(9.6-20.8)\end{array}$ & $\begin{array}{l}1.8 \\
(0-3.8)\end{array}$ & $\begin{array}{l}5.9 \\
(2.1-9.6)\end{array}$ \\
\hline T1G3 & $\begin{array}{l}10.6 \\
(6.5-14.7)\end{array}$ & $\begin{array}{l}18.9 \\
(13.6-24.4)\end{array}$ & $\begin{array}{l}11.4 \\
(5.3-17.5)\end{array}$ & $\begin{array}{l}19.8 \\
(12.0-27.6)\end{array}$ \\
\hline
\end{tabular}
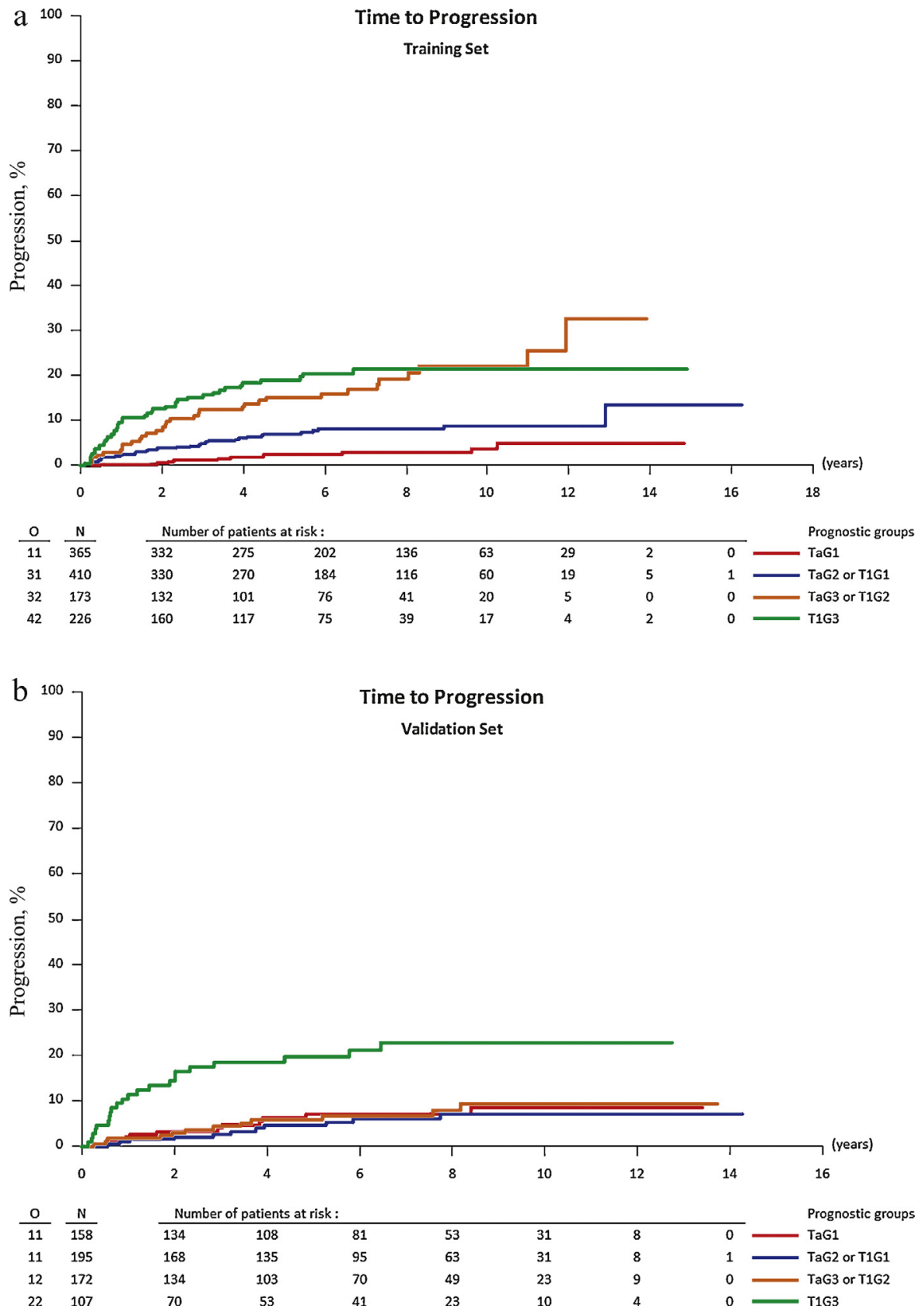

Fig. 2 - Time to progression according to prognostic groups: (a) training set; (b) validation set. 
Table 6 - Probabilities of death due to bladder cancer and 95\% confidence intervals in the training and validation datasets according to stage and grade

\begin{tabular}{|c|c|c|c|c|}
\hline & \multicolumn{2}{|c|}{ Training data set: $\mathrm{C}$-index 0.72} & \multicolumn{2}{|c|}{ Validation data set: $\mathrm{C}$ index 0.71} \\
\hline & $1 \mathrm{yr}, \%(95 \% \mathrm{CI})$ & 5 yr, \%(95\% CI) & $1 \mathrm{yr}, \%(95 \%$ CI) & $5 \mathrm{yr}, \%(95 \% \mathrm{CI})$ \\
\hline TaG1 & $0:$ no events & $\begin{array}{l}0.87 \\
(0-1.9)\end{array}$ & 0: no events & $\begin{array}{l}1.5 \\
(0-3.6)\end{array}$ \\
\hline TaG2 or T1G1 & $\begin{array}{l}0.75 \\
(0-1.6)\end{array}$ & $\begin{array}{l}3.6 \\
(1.7-5.6)\end{array}$ & $0:$ no events & $\begin{array}{l}1.7 \\
(0-3.6)\end{array}$ \\
\hline TaG3 or T1G2 & $0:$ no events & $\begin{array}{l}7.1 \\
(3.1-11.2)\end{array}$ & $0:$ no events & $\begin{array}{l}1.4 \\
(0-3.3)\end{array}$ \\
\hline T1G3 & $\begin{array}{l}1.9 \\
(0.05-3.7)\end{array}$ & $\begin{array}{l}11.5 \\
(6.9-16.0)\end{array}$ & $\begin{array}{l}4.8 \\
(0.70-8.9)\end{array}$ & $\begin{array}{l}11.3 \\
(5.0-17.6)\end{array}$ \\
\hline
\end{tabular}
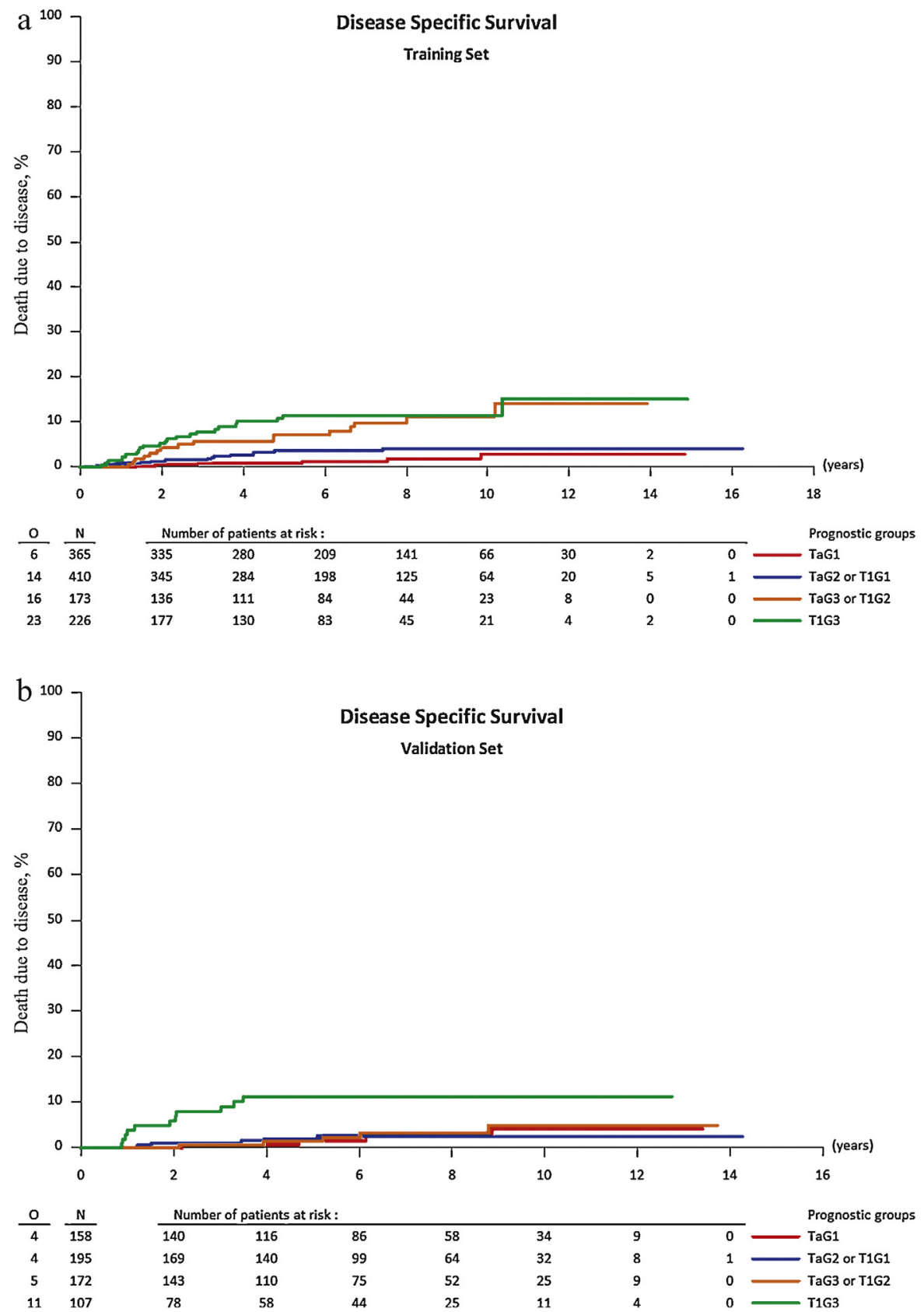

Fig. 3 - Disease-specific survival according to prognostic groups: (a) training set; (b) validation set. 


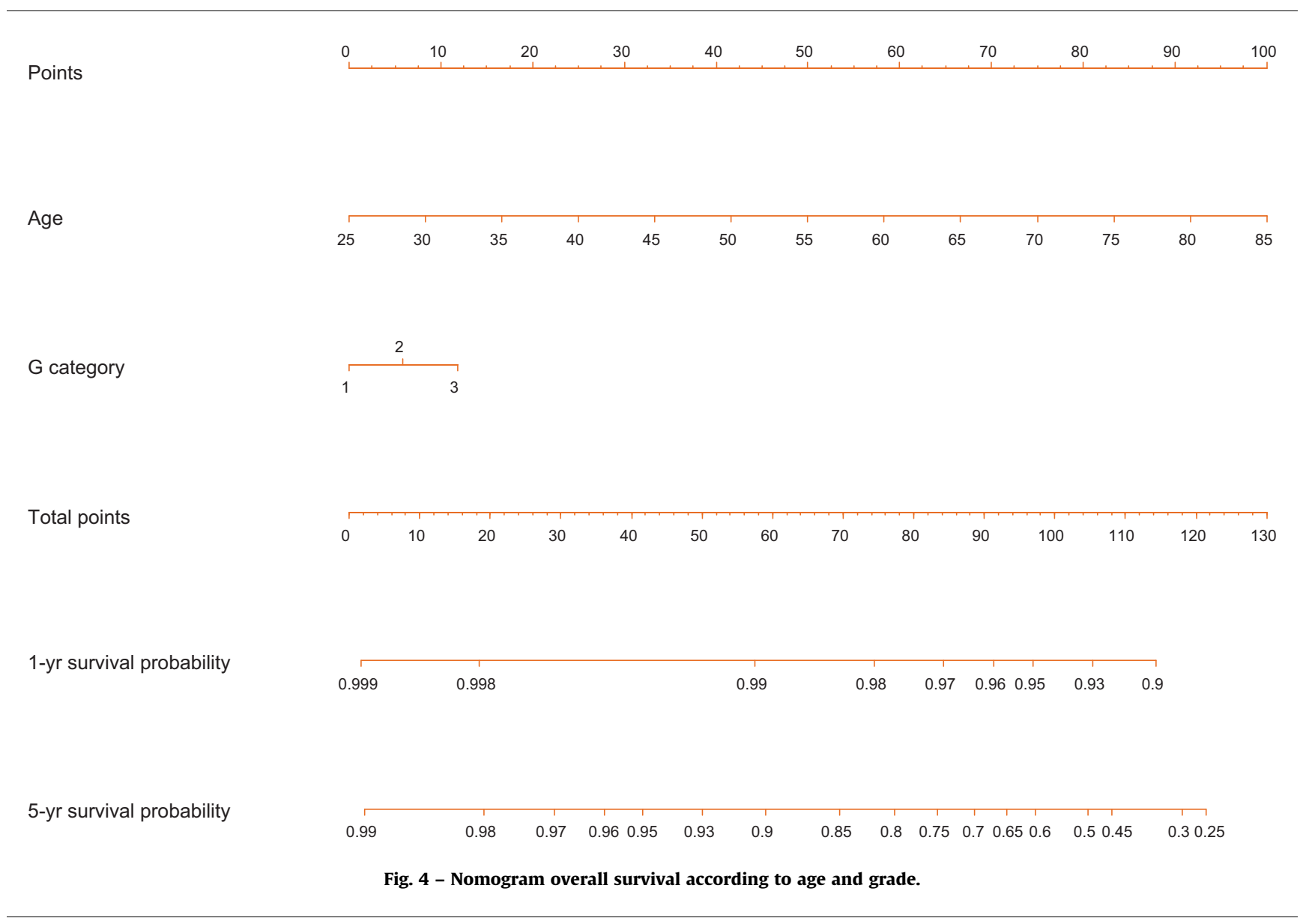

and CUETO. As expected, T1G3 patients had the worst prognosis with 1 - and 5-yr progression rates of $11.4 \%$ and $19.8 \%$, respectively.

CUETO models for recurrence and progression are "optimized" for their data set and do not perform as well when applied to EORTC data. The longer duration of BCG in EORTC studies may have also had an impact on the results.

For the first time, prognostic factor analyses for both DSS and OS are provided. For DSS, the most important factors were the same as for progression: stage and grade. T1G3 patients had the worst prognosis with 1- and 5-yr diseasespecific death rates of $4.8 \%$ and $11.3 \%$, respectively. Although grade remained statistically significant for OS, age was the dominant factor (Fig. 4). The clinical utility of this nomogram in daily practice still needs to be proven.

Patients previously identified at the highest risk of recurrence $[3,5,6]$ still remain at high risk even when treated with 1-3 yr of maintenance BCG. Patients with four or more tumors and a prior recurrence rate of more than one per year have a probability of recurrence at 1 and $5 \mathrm{yr}$ of $33.0 \%$ and $51.7 \%$, respectively, even if no tumor was detected at the first follow-up cystoscopy. With recurrences after $>10 \mathrm{yr}$, our study along with studies from CUETO, SWOG, and FinnBladder show the need for long-term follow-up in NMIBC patients [5,6,16,17].

Many patients do well on maintenance, with a 5-yr recurrence-free rate of $58.7 \%$. Although we can accurately identify patients who do not progress or die due to their disease (approximately 95\% of TaG1 patients do not progress, and $98 \%$ do not die due to their disease), we cannot reliably identify the important subgroup of patients who do progress and die of their disease. Patients with T1G3 tumors, even without CIS, do relatively poorly, with 5-yr progression- and disease-specific death rates of $19.8 \%$ and $11.3 \%$, respectively, but we need improved methods to identify the really high-risk patients and develop effective treatments for them.

Because the number of tumors and prior recurrence rate have also been shown to contribute to a worse prognosis, T1G3 patients with recurrent and/or multiple lesions should be considered for early cystectomy instead of BCG, especially if they have concomitant CIS, as concluded by Gontero et al [18]. However, we need better markers of immune response and molecular profiling to optimize patient selection and treatment strategy [19,20].

This data set has a number of limitations. No patients with CIS were included. Both studies were carried out when routine repeat transurethral resection was not performed in high-risk patients. This could have contributed to understaging that may have led to higher progression rates, especially in T1G3 patients. There was no central pathology review. No information was collected on the status of the upper urinary tract upon recurrence or progression or on potential biomarkers. It was not possible to make a meaningful distinction between $1 \mathrm{yr}$ and $3 \mathrm{yr}$ of maintenance in the analysis. Nevertheless, this study provides the 
best available information about the prognosis of patients treated with the currently recommended 1-3 yr of maintenance BCG.

\section{Conclusions}

NMIBC patients treated with 1-3 yr of maintenance BCG have a heterogeneous prognosis for both time to first recurrence (according to the prior recurrence rate and number of tumors) and time to progression and death due to BCa (based on tumor stage and grade).

Patients at high risk of recurrence and/or progression still do poorly on current maintenance schedules. Alternative treatments are urgently required.

Author contributions: Richard J. Sylvester had full access to all the data in the study and takes responsibility for the integrity of the data and the accuracy of the data analysis.

Study concept and design: Sylvester, Cambier, L. Collette. Acquisition of data: Gontero, Brausi, van Andel, Kirkels, Calais Da Silva, Oosterlinck, Prescott, Kirkali, Powell, de Reijke, Turkeri, Oddens. Analysis and interpretation of data: Sylvester, Cambier, L. Collette. Drafting of the manuscript: Sylvester, Cambier, L. Collette, Oddens. Critical revision of the manuscript for important intellectual content: Cambier, Sylvester, L. Collette, Gontero, Brausi, van Andel, Kirkels, Da Silva, Oosterlinck, Prescott, Kirkali, Powell, Reijke, Turkeri, S. Collette, Oddens.

Statistical analysis: Sylvester, Cambier, L. Collette. Obtaining funding: Sylvester, L. Collette.

Administrative, technical, or material support: None.

Supervision: Sylvester, L. Collette.

Other (specify): None.

Financial disclosures: Richard J. Sylvester certifies that all conflicts of interest, including specific financial interests and relationships and affiliations relevant to the subject matter or materials discussed in the manuscript (eg, employment/affiliation, grants or funding, consultancies, honoraria, stock ownership or options, expert testimony, royalties, or patents filed, received, or pending), are the following: None.

Funding/Support and role of the sponsor: The Fondation Contre le Cancer and Kom op tegen Kanker provided support to the EORTC to design and conduct the study; collect, manage, analyze, and interpret the data; and prepare, review, and approve the manuscript.

Acknowledgments: We are grateful to the Fondation Contre le Cancer and Kom op tegen Kanker for providing core support to the EORTC through the EORTC Charitable Trust.

\section{Appendix A. Supplementary data}

Supplementary data associated with this article can be found, in the online version, at http://dx.doi.org/10.1016/j. eururo.2015.06.045.

\section{References}

[1] Babjuk M, Burger M, Zigeuner R, et al. EAU guidelines on nonmuscle-invasive urothelial carcinoma of the bladder: update 2013. Eur Urol 2013;64:639-53.

[2] Babjuk M, Böhle A, Burger M, et al. Guidelines on non-muscleinvasive bladder cancer (Ta, T1 and CIS). European Association of
Urology Web site. http://uroweb.org/guideline/non-muscle-invasivebladder-cancer/.

[3] Sylvester RJ, van der Meijden APM, Oosterlinck W, et al. Predicting recurrence and progression in individual patients with stage Ta T1 bladder cancer using EORTC risk tables: a combined analysis of 2596 patients from seven EORTC trials. Eur Urol 2006;49: 466-77.

[4] Fernandez-Gomez J, Madero R, Solsona E, et al. The EORTC tables overestimate the risk of recurrence and progression in patients with non-muscle-invasive bladder cancer treated with bacillus Calmette-Guérin: external validation of the EORTC risk tables. Eur Urol 2011;60:423-30.

[5] Fernandez-Gomez J, Solsona E, Unda M, et al. Prognostic factors in patients with non-muscle-invasive bladder cancer treated with bacillus Calmette-Guérin: multivariate analysis of data from four randomized CUETO trials. Eur Urol 2008;53:992-1002.

[6] Fernandez-Gomez J, Madero R, Solsona E, et al. Predicting non muscle invasive bladder cancer recurrence and progression in patients treated with bacillus Calmette-Guérin: the CUETO scoring model. J Urol 2009;182:2195-203.

[7] Rosevear HM, Lightfoot AJ, Kenneth G, Nepple KG, O'Donnell MA. Usefulness of the Spanish Urological Club for Oncological Treatment scoring model to predict non muscle invasive bladder cancer recurrence in patients treated with intravesical bacillus CalmetteGuérin plus interferon- $\alpha$. J Urol 2011;185:67-71.

[8] Sylvester RJ, Brausi MA, Kirkels WJ, et al. Long-term efficacy results of EORTC Genito-Urinary Group randomized phase 3 study 30911 comparing intravesical instillations of epirubicin, bacillus CalmetteGuérin and bacillus Calmette-Guérin plus isoniazid in patients with intermediate and high-risk stage Ta T1 urothelial carcinoma of the bladder. Eur Urol 2010;57:766-73.

[9] Oddens J, Brausi M, Sylvester R, et al. Final results of an EORTC-GU Cancers Group randomized study of maintenance bacillus CalmetteGuérin in intermediate and high risk Ta, T1 papillary carcinoma of the urinary bladder: one-third dose versus full dose and 1 year versus 3 years of maintenance. Eur Urol 2013;63:462-72.

[10] Schemper M, Smith TL. A note on quantifying follow-up in studies of failure time. Controlled Clin Trials 1996;17:343-6.

[11] Miao Y, Cenzer IS, Kirby K, Boscardin WJ. Estimating Harrell's optimism on predictive indices using bootstrap samples [paper 504-2013]. SAS Global Forum 2013. SAS Web site. http://support. sas.com/resources/papers/proceedings13/504-2013.pdf.

[12] Kuk D, Varadhan R. Model selection in competing risks regression. Stat Med 2013;32:3077-88.

[13] Harrell FE, Lee KL, Mark DB. Multivariable prognostic models: issues in developing models, evaluating assumptions and adequacy, and measuring and reducing errors. Stat Med 1996;15: 361-87.

[14] Yan G, Greene T. Investigating the effects of ties on measures of concordance. Stat Med 2008;27:4190-206.

[15] Brausi M, Collette L, Kurth K, et al., EORTC Genito-Urinary Tract Cancer Collaborative Group. Variability in the recurrence rate at first follow-up cystoscopy after TUR in stage Ta T1 transitional cell carcinoma of the bladder: a combined analysis of seven EORTC studies. Eur Urol 2002;41:523-31.

[16] Lamm DL, Blumenstein BA, Crissman JD, et al. Maintenance bacillus Calmette-Guerin immunotherapy for recurrent Ta-T1 and carcinoma in situ transitional cell carcinoma of the bladder: a randomized Southwest Oncology Group Study. J Urol 2000;163:1124-9.

[17] Jarvinen R, Marttila T, Kaasinen E, et al. Long-term outcome of patients with frequently recurrent non-muscle-invasive bladder carcinoma treated with one perioperative plus four weekly instillations of mitomycin $C$ followed by monthly bacillus CalmetteGuérin (BCG) or alternating BCG and interferon- $\alpha 2 b$ instillations: 
prospective randomized FinnBladder-4 study. Eur Urol 2015;68: $611-7$.

[18] Gontero P, Sylvester R, Pisano F, et al. Prognostic factors and risk groups in T1G3 non-muscle-invasive bladder cancer patients initially treated with bacillus Calmette-Guérin: results of a retrospective multicenter study of 2451 patients. Eur Urol 2015;67: $74-82$.
[19] Zuiverloon TCM, Nieuweboer AJM, Vekony H, Kirkels WJ, Bangma $\mathrm{CH}$, Zwarthoff EC. Markers predicting response to bacillus CalmetteGuérin immunotherapy in high-risk bladder cancer patients: a systematic review. Eur Urol 2012;61:128-45.

[20] Biot C, Rentsch CA, Gsponer JR, et al. Preexisting BCG-specific T cells improve intravesical immunotherapy for bladder cancer. Sci Transl Med 2012;4:137ra72. 